\title{
Modern Approaches to Consumer Markets Segmenting
}

Jury Davydovich Schmidt

\author{
Far Eastern Federal University, Russia, 690922, Vladivostok v. Ayaks, Russkii Island, Russia
}

Natalya Stepanovna Martyshenko

Vladivostok state university economy and service, Russia, 690014, Vladivostok, street Gogol, 41

\section{Doi:10.5901/mjss.2015.v6n5p367}

\section{Abstract}

The variety of tasks of segmentation and conditions of markets formation generated a variety of methods of segmentation. The aim of the article is to create a scientific guide in the space of modern methods and tools for consumer markets segmenting. Among the methods of segmentation the most powerful tools are methods of multivariate data classification. The article discusses different approaches for constructing multivariate classification algorithms. During the study of socio-economic systems, the vast majority of events are not measured directly (mental abilities, personality traits, tolerance, competence, mobility, political beliefs, etc.). In such cases it is advisable to use the latent pattern or latent structural analysis. In the practice of segmentation we often have to deal with qualitative data. Processing qualitative data is based on the principles of typologies, which are embodied in computer technology. The use of a combination of qualitative and quantitative methods is often the best solution to the problem of market segmentation.

Keywords: market segmentation, multivariate data analysis, latent structural analysis, analysis of qualitative data

\section{Introduction}

The main impetus for the development of any market is a buyer. Many buyers are uniform. They differ in tastes, desires and motives of shopping. This phenomenon originated such an important tool of markets research, as market segmentation, whose goal is to develop a flexible policy of engagement with different groups of consumers.

At present there are many approaches of market segmentation, in which it is difficult to navigate by practitioners. Therefore, in the work there was an attempt to systematize the methods and tools of consumer markets segmenting.

Market segmentation is a formal procedure based on the use of multivariate statistical analysis of the results of marketing research.

The objects of market segmentation are consumer groups; group of products or services; competing factories.

Goals of market segmentation:

- Meeting the needs of customers in a variety of products, reduction of goods and services in accordance with the wishes and preferences of consumers;

- The development of an effective marketing strategy, based on an understanding of the behavior of potential buyers;

- optimization of companies costs for the development, production and sale of goods;

- Improving the efficiency and adequacy of the decisions, the degree of their validity by providing managers with the necessary information about the current situation and expectations for the future;

- Improving the competitiveness of the goods and gain competitive advantages of goods;

- Preparation for the transition to the untapped market segments and new markets;

- Formation of scientific and technical policy of the company in accordance with the growth of consumer demand.

The variety of tasks and conditions of formation of segmentation markets has generated a lot of segmentation methods [1]. Currently, in the market research practice following methods of segmentation became widespread:

- The method of multi-dimensional classification;

Methods of archetype segmentation;

- Method of segmentation on benefits;

- Method of meshing segmentation 
- Method of grouping;

- The method of functional maps;

- Method of segmentation based on Abel matrix;

- Method of segmentation of consumers according to their degree of loyalty;

- Method of segmentation on benefits;

Among the methods of segmentation, which appeared quite recently, you can see the following:

- Method of collaborative filtering;

- Method of latent models;

- Method of flexible segmentation;

- Method of componential segmentation.

In scientific journals in marketing publications are constantly emerging that deal with all new methods and approaches to segmenting consumer markets $[2,3,4,5]$.

The aim of the study is to create a scientific guide in the space of modern methods and tools for consumer markets segmenting.

\section{Methods Used for Segmentation}

Space of methods of segmentation becomes more obvious if we mark certain common approaches that are used in the development of the individual methods of segmentation. We found three approaches. However, such a division is quite arbitrary, since in the solution of specific problems approaches may overlap.

\subsection{The use of methods of multi-dimensional classification}

Among the methods of segmentation the most powerful tool are methods of multivariate data classification. Application of the multi-dimensional classification is called cluster analysis.

Cluster analysis is a set of methods to classify multi-dimensional observation, each of which is described by a set of input variables $X_{1}, X_{2}, \ldots, X_{j}, \ldots, X_{m}$. The goal of cluster analysis is the formation of groups of similar objects, which are called clusters.

Classification problem should be distinguished from the task group. The task group is the group where the first data is divided by one characteristic level, followed by another characteristic level, etc. In contrast to the task of grouping in the cluster analysis formation of groups of objects (classes) is made with all the grouping characteristics simultaneously.

To solve the problem of classification it is necessary to introduce the concept of similarity of objects for the observed characteristics. Objects in each class that have a certain degree of similarity should be get in one class.

To quantify the similarity metric concept is introduced. The similarity between the objects will be determined depending on the distance of the selected metric space. If the object described by $m$ attributes is represented by a point in the m-dimensional space, the similarity of objects with each other will be defined as the distance in this metric space.

\subsection{In the cluster analysis a wide variety of ways to measure the distances (metrics) are used.}

An example of one of the most common metrics of similarity is Euclidean distance.

To solve the problem of classification it is necessary to consider the distance between each pair of objects. The distances between pairs of objects are reduced to a similarity matrix. This is a symmetric matrix. Diagonal matrix arranges zero.

To assess the similarity of objects using distance measures is useful when using numeric attributes. But often there are attributes that are measured at other scales (for example, rank, or, in general, nominal). In this case, all the attributes needed to classify are transformed to a representation in binary code. So, each object is described by the vector $\overline{X_{i}}=\left(x_{i 1}, x_{i 1}, \ldots x_{i m}\right) i=\overline{1, n}$, each component of which takes the values 0 or 1 . Examples of similarity measures in binary scale factor measurements can be Rao and Hamman coefficients. The advantage of the binary representation is the possibility of a joint analysis of the measured traits in different scales.

Another important variable in the cluster analysis is the distance between the whole groups of objects. The most common methods to determine the distance between the clusters are: the method of "nearest neighbor", method of "farthest neighbor", method for estimating the distance between the centers of gravity. 
Among all the methods of classification most common are hierarchical agglomerative methods. The basic idea of these methods is that the first step of each object is treated as a separate cluster. Hierarchical procedure is a stepwise combine of the most similar classes. The similarity of the classes is estimated by the matrix of distances or similarity matrix. In the first step similarity matrix has a dimension $n \times n$. In the next step when the two classes are combined similarity matrix is recalculated. The dimension of the matrix is reduced by one and becomes $\left[{ }^{(n-1) \times(n-1)}\right]$. The process is completed in $n-1$ steps, when all objects are combined into one class. The process of combining objects can be represented as a graph tree (dendrogram). Dendrogram indicates the numbers of combined objects and the distance at which there was an association. When you select a class you guide the jumps of similarity metric in the dendrogram.

A method of multi-dimensional classification is widespread in the practice of economic analysis. It is known as "kmeans". It was proposed by McQueen. This method relates to the group of iterative methods of classification. There are many modifications of this method. Consider the classification algorithm in its original form.

Suppose there are $\mathrm{n}$ objects (cases), each of which is characterized by characteristics $\mathrm{m} X_{1}, X_{2}, \ldots, X_{j}, \cdots, X_{m}$. It is necessary to break the observations of a number of classes $k$.

Step $0 \mathrm{n}$ points from study population $\mathrm{k}$ points are randomly selected. These points are taken as the centers of classes.

Iteration. The set of points is divided into $\mathrm{k}$ classes at a minimum distance from the centers of classes. To calculate the distance you can use any metric. The most commonly Euclidean distance is used. There is a recalculation of class centers as centers of gravity of the points attached to classes.

Check. If the centers of classes during the next iteration are not changed, the classification process is completed; otherwise we proceed to the "iteration".

There is a large group of algorithms for multi-dimensional classification based on the application of graph theory. Representative of this group is the algorithm "Crab" [6]. On similar principles group of algorithms FOREL is organized.

Let's study the principles of the algorithm "Crab". The algorithm begins by finding pairs of points with a minimum distance between them. These points are connected by an edge of the graph. Then the next closest point of the numbers is connected from not attached to the already constructed part of the graph. This procedure is repeated until all the points will not yet be connected by edges of the graph.

Such graph will have no loops, and the total length of all its edges is minimal. Graph with such properties is called open-shortest path. To partition the set of points on the graph into the two we break the longest edge. Then the process is repeated until the appropriate structure of the classes. Algorithm "Crab" has many modifications. a new concept $\lambda$ distance and quality criteria of the partition is introduced for further development of the algorithm.

In the study of socio-economic systems, the vast majority of events are not measured directly (mental abilities, personality traits, tolerance, competence, mobility, political beliefs, etc.), which led to the emergence of a new concept "latent" variables. For the study of latent variables latent models or latent structural analysis are used. It is a fairly broad class of models that have been applied in various fields, including solving segmentation problems.

Analysis of latent structure (from Lat. Latentis - hidden, invisible) - is a statistical analysis of empirical data, allowing for respondents' answers to a set of questions to identify their distribution in some hidden (latent) characteristic. This feature cannot be measured directly, but the use of a variety of issues can fix its various manifestations. The method was proposed by the famous American sociologist Paul Lazarsfeld [7].

The essence of the model proposed by Lazarsfeld, was as follows. It is assumed that there is a latent variable that explains the external behavior of the respondents. This behavior can be explained by analyzing the responses of each person on certain dichotomous questions of the questionnaire. Latent variable is nominal, the number of its values researcher knows beforehand. The explanatory power of latent variable is caused by the fact that it is the cause of the link between the observed variables $[8,9]$.

At the heart of the classical latent structural analysis there is a fundamental axiom of Lazarsfeld about local independence: the fixation of values of latent variable connection between the observed variables disappears.

Subcombination of the respondents with the same values of latent variable forms latent classes. There are several types of latent models: models for continuous latent variable; models for discrete latent variable; model for dichotomous features.

The advantage of latent structural analysis compared with analysis by the cluster is that this method allows analysis to include variables measured in different scales.

One of the approaches to the estimation of latent variables is to use the Rasch model.

Measurements using a model Rasch - is the process of transforming raw data in the form of the test into interval scale of natural logarithms. To calculate the latent variables in the model Rasch the concept of "logit" is introduced. 
Logit is a standard unit, easily bred in any other scale. Because the Rasch scale is interval, this permits to use a large number of various statistical analysis procedures. In addition, in interval scale zero reference point (0) is not fixed, so the indicators in logit transferred to the other system, for example, points through linear transformations. And as a starting point in the logit system it is most appropriate to use average values of observed variables.

Initially, the Rasch model was used to analyze the knowledge in testing. Currently, the scope of the model applies to other objects. For example, the unit of Rasch model can be applied to structural analysis of these questionnaires, including questions that characterize the tolerance to different socio-economic processes. Tolerance can be expressed in the choice of answers listed below:

- Strong agreement (of course yes);

- Weak agreement (more likely than not);

- Slight disagreement (more no than yes); strong opposition (of course not).

This model allows to measure in the same scale (logit) the level of tolerance and inclusiveness of the items of the questionnaire [10].

1.2. The use of graph theory and graphic images

Segments methods based on graph theory are successfully used to identify market. Examples of this approach can be found in $[11,12]$. The advantage of this approach is in the visualization of the segments that facilitates meaningful analysis.

The mathematical model of market segmentation services is based on a 3-partite 3-uniform hypergraph $G=(V, E)=\left(V_{1}, V_{2}, V_{3}, E\right)$. The tops of the first part, i.e. $v \in V_{1}$, they correspondence with the elements of the set provided by the company or group of companies services. Each top of the second part $v \in V_{2}$ uniquely corresponds to an element of the plurality of users, classified by demographic characteristics. Third part tops $v \in V_{3}$ are correspondence with the elements of the set of consumers, classified on the basis of "the level of income": low, below average, average, above average, high, elite class. To construct a set of edges $E=\{e\}$ all possible triples of tops are considered $v_{1}, v_{2}, v_{3}$ $v_{1} \in V_{1}, v_{2} \in V_{2}, v_{3} \in V_{3}$. Every such triple is called admissible if $v_{1}$ service may be acceptable to consumers of this level of income $v_{3}$ for this demographic category ${ }^{v_{2}}$. A set of all edges ${ }^{E=\{e\}}$ is defined as the set of all admissible triples $e=\left(v_{1}, v_{2}, v_{3}\right), v_{i} \in V_{i}, i=\overline{1,3}$.

In the Hypergraph $G=(V, E)=\left(V_{1}, V_{2}, V_{3}, E\right)$ feasible solution of the problem of market segmentation services is just a such its subhypergraph $x\left(V_{x}, E_{x}\right), V_{x} \subseteq V, E_{x} \subseteq E$, in which every connected component is a single star with center at the top $v^{V \in V_{1}}$. Through $X=X(G)=\{x\}$ we set all feasible solutions of the problem of hypergraph coverage by $\mathrm{G}$ stars. For example, one of the solutions is presented in Fig. 1.

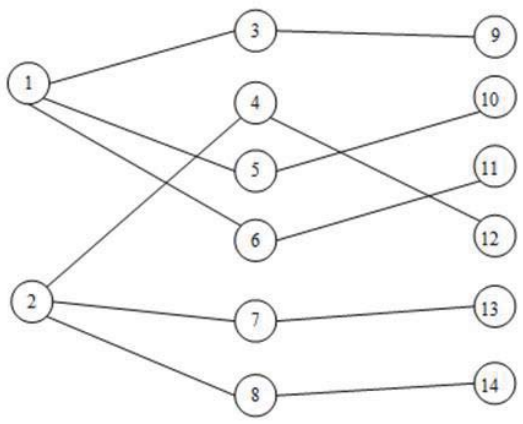

Figure 1. Allowable Floor of graph with stars

For the numerical evaluation of the quality of feasible solutions to each edge $e \in E$ of the hypergraph ${ }^{G=(V, E)}$ three weights $w_{v}(e), v=\overline{1,3}$ are attributed, which are the expert evaluations. As the weights may be: the power of positioning services, the expected stability of positioning and others. Quality of valid solution to this problem is estimated using a vector objective function (1): 
$F_{v}(x)=\sum_{e \in E_{x}} w_{v}(e) \rightarrow \max , v=\overline{1,3}$

Recently method of segmentation based on collaborative filtering is becoming more common $[13,14]$.

In general, the collaborative filtering algorithm can be described as follows: there are many users $\left\{u_{1}, u_{2}, \ldots u_{n}\right\}$ and many objects $\left\{b_{1}, b_{2}, \ldots b_{m}\right\}$. Each user has a list of valued by him objects. Assessment may belong to different scale from 1 to 10,1 to 5 , and so on, as well as different types of scales: ordinal or relative. If user $u_{i}$ wishes to get a recommendation (or his assessment forecast on an invaluable by him object), then by the known assessments a plurality of users, who a similar in preferences (or by assessments on the same objects) are found. Then the algorithm makes recommendations to the user ${ }^{u_{i}}$ (or calculates predictive assessment of the object), based on the assessments similar to $u_{i}$ users by their preferences.

\subsection{Market segmentation by psychographic characteristics}

Methods of market segmentation by psychographic characteristics are becoming more common. Market segmentation by psychographic characteristics is the process of separating all buyers into homogeneous groups according to criteria such as values, beliefs, motivation for buying goods and personality type.

Psychographic segmentation characteristics are based on the theory of typological analysis.

Typological analysis is a metamethod of data analysis, a set of methods for the study of social phenomena, allowing to highlight socially significant, internally homogeneous, qualitatively distinct groups of empirical objects characterized by type forming signs which nature is different, and interpreted as the existence of different types of media phenomenon [15].

In world literature there is a term "qualitative research methods", which is sometimes called "soft" as opposed to "quantitative" and "hard". In a certain sense we can also speak of formalized or weakly formalized approach in comparison with a rigidly formalized [16, 17, 18].

Typology base is a set of propositions (statements) of the proximity (similarity) objects' carriers of data about the studied social phenomena (processes).

The subject of the typology is a collection of the main characteristics of a social phenomenon that is responsible for the assignment of empirical objects to the same type of group.

Consider some of the terms that are used in the typological analysis.

Type - the form of the existence of social phenomena in science or in everyday life. Type - the essence, the knowledge of which is always relative. It has three main values in the sense - standard, typological, typical.

Typological - in typological analysis: special, general, combining.

Typization - construction by people a social reality on the basis of giving to others labels, spontaneous classification.

Typologization - procedure of systematization of knowledge about the studied phenomena or for administration (defining) types, or to seek knowledge about types. Tipologization is used to construct types.

Typology - the collection of types, the result of their construction.

Method of type's construction.

Type forming feature is a characteristic, feature of social phenomena, based on which types are constructed or formulated the hypothesis of their existence. Type forming feature is a conceptual variable.

There are several approaches to solve the problem of unstructured and non-formalized data. One of the main tasks is to develop an algorithm for construction typologies so as to overcome the subjectivity of the researcher, do not miss the important information. The algorithms involve the use of compression and structuring of information so that it has retained the properties of the object.

We can distinguish three approaches for constructing typologies:

- The concept of typological operations of A. Barton and P.F. Lazarsfeld;

- Analysis of the structure of W. Gerhard;

- Typological analysis of W. Kukaritsa.

The first approach is based on a "typological operations": reduction, substructure and transformation. By identifying features and intensity degree the space of properties is being built that is the basis of the typology. Using graphical and tabular presentation of the data, all possible combinations and all potential types are determined. All types are linked to a single space. Combinations of features can be reduced. Substructure reveals signs of the space itself, the basis of 
typology that can be transformed in the interpretation of constructed types. Despite the fact that this approach has been proposed for the construction of types of quantitative research strategy, it is central to the generalization of non-numerical information.

The second and third approaches use the same basic typological operations while trying to overcome its main drawback: the definition of criteria for selection of features for data analysis. In the second approach there are "ideal types" that serve as a basis for the analysis of the information obtained in the course of the study. In the first stage there is a comparison of cases through their reconstruction, to identify their characteristics. This brings to the study the transparency of generalization process and its results. In the second step studied cases are grouped using their comparison. These techniques are broadly consistent of "typological concept of operations" and let you know all the potentially possible combinations of features. At the last stage the meaning relations within and between obtained groups are identified and explained. For this purpose, an assay of structure and process was developed, consisted of two-step synthesis. The main disadvantage of this approach is the difficulty to abstract from the subjective perceptions of the researcher in the generalization of data and lack of verification algorithm of constructed types.

Typological analysis in this approach has a number of features compared with other methods of generalization of qualitative data. In the process of abstracting from each individual investigated case and produce a typical event, as a result of the ordered phase of sequence. "Structural hermeneutics", on the contrary, understands the material in its unit, not divided to a particular investigational case. Typological analysis is the point of intersection between the individual and the common history. In the second approach, the case studies are saved as much as possible in its entirety, in the third approach in the analysis of individual cases and their compare a thematic synthesis is used.

The third area can not be fully considered as a separate entrance. It is a tool built for the purpose of expressing a methodological opinion of $\mathrm{M}$. Weber. In developing tools of typological analysis (software) there was made an attempt to connect the different ways of typologization of non-numerical information with regard to their advantages and disadvantages.

Famous sociologists N. Fielding and R. Lee in objective area of tool analysis of qualitative data suggested the use of a special term "computer, but assisted analysis of qualitative data» (Computer Assisted Qualitative Data AnalysiS, CAQDAS). Modern computer-assisted analysis of qualitative data is a methodological research area joining scientists in many countries. Assisted analysis is presented by a variety of software packages, including: Atlas.ti, MAXQDA, NVivo, xSight, Qualrus, Ethnograph and others. These packages are a class of computer programs, which include in its architecture special structures called encoding functions and reconstruction of high-quality, non-numerical data (coding and retrieval functions). Encoding function and reconstructing the data is a computer tool, used in man-machine mode and assists the user in the study of the data presented in the so-called non-numeric formats. At the heart of the assisting there is a unit of analytical relabeling, called codes, and the introduction of binding to each other which is carried by the user. A more detailed analysis of the methodological development of computer tools qualitative data analysis is presented in [19].

Noting the opportunities of foreign computer tools of qualitative data analysis it should be noted that they did not find their spread not only among Russian scientists dealing with market segmentation, but also among Russian sociologists. These tools have a slightly different focus and more designed to solve the humanitarian and linguistic problems. For practitioners involved in market segmentation easy-to-use and affordable means of analyzing of qualitative information are needed.

\section{Results}

\subsection{The method of analysis of qualitative data, developed by the authors}

The methodology developed by the authors, includes quite easy to use computer software tools that can be used within the computer environment EXCEL, which is the most common among market researchers. The results of use of the methodology of analysis of qualitative data, which are formed for the purpose of market research and, in particular, the tourist market, are presented in [20, 21].

The proposed methodology is based on a new approach of the initial submission of quality information. It is believed that the initial information, in whatever form it was not going to, is presented in the table "object property". Apart from the usual features that allow only one value of the investigated properties, we allow in the table "object property" component features. The main symptom occurs when answering the question the respondent can specify several answers. For example, when responding to the survey question: "What are the major cities you visited in the last three years," the respondent can name several cities. Thus, the component feature consists of a few simple answers. To 
identify the composite sign, we introduce a single unique separator character. The simple answer may consist of several words, or even can be formulated in the form of a proposal.

The transition from an unstructured presentation of information to a structured, allowed quantitative processing is performed by the operation of typization.

Operation of typization - it's a simple replacement of the original statement (in text form) to close or similar in value, or generalizing statement (in the form of text). During typization operation auxiliary table - "the list of characteristic values" is formed. The main columns of this table include all the unique values of the original simple composite feature. That is, the table does not contain duplicate values. Apart from the main column of the table "List of characteristic values" has a column in which the frequency encountered simple values are calculated. Not the basic data table, but auxiliary, is getting typization which is clearer than the original data table. At the end of the cycle typizations an adjustment of the main table of data is made. Scheme of computer unstructured data processing is shown in Fig. 2.

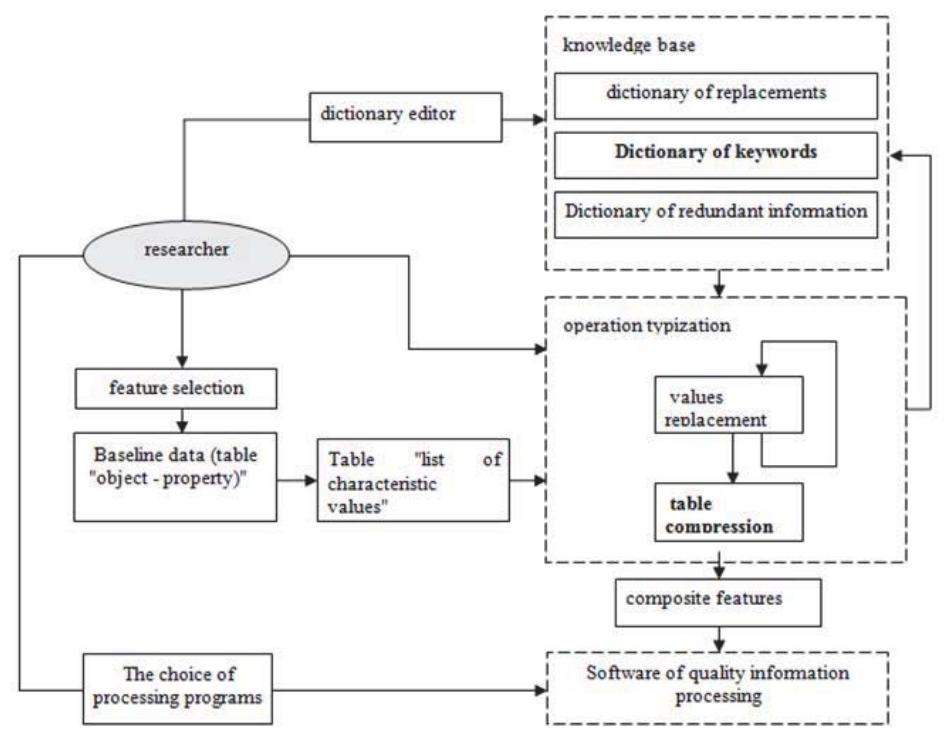

Figure 2. Scheme of a computer unstructured data processing technology.

Improving the efficiency of computer processing technology of high-quality data is achieved through the creation and use of knowledge base. Computer technology, allowing to use the knowledge base, belong to a class of expert systems. The main distinguishing feature of the expert system is the ability to make correct predictions. Produce all kinds of prompting the user during operation, special software can significantly reduce the time of the user work.

Prompting the user is carried out with the help of special dictionaries. Formation of dictionaries is made in the process of the user on the task of typization. Dictionaries accumulate user experience acquired during the process of solving problems of typization of qualitative attributes. All dictionaries are stored in an Access database file

The considered technology has a wide approbation. It has been used for the processing of more than ten different questionnaires.

\section{Conclusion}

Methodology for analyzing data used in the segmentation should be seen not so much as the study of methods, but as the doctrine of the interaction methods with each other on different classes of research practices analysis. Structurally, this methodology includes techniques and methods of data collection and measurement information, and mathematical methods.

Most of researchers use in their practice very limited set of segmentation tools. This is due both to insufficient knowledge of the theoretical foundations of various methods of segmentation and available software. 
Using a combination of qualitative and quantitative methods is often the best solution to the problem of market segmentation. Various methods complement and monitor each other; limitations of one method are balanced by the other.

Possession of a wide range of segmentation tools removes many of the restrictions on the wording of questions asked by users, and allows you to extend the range of tasks of the meaningful analysis of the selected segments. Therefore practitioners must constantly expand the range of tools of segmentation that they use.

\section{References}

Hassan, S. S., \& Craft, S. (2012). Examining world market segmentation and brand positioning strategies. Journal of Consumer marketing, 29(5), 344-356

Blythe, J. (2013). Principles and practice of marketing. Sage.

Gamble, P. R. (2010). Marketing revolution. Strategic Direction, 26(4).

Goyat, S. (2011). The basis of market segmentation: a critical review of literature. European Journal of Business and Management, 3(9), 45-54.

McDonald, M. (2012). Market segmentation: How to do it and how to profit from it. John Wiley \& Sons.

Zagoruiko, N.G. (1999). Applied methods of data analysis and knowledge (p. 270). Novosibirsk: Publishing House of the Institute of Mathematics.

Balazova, E. \& Marsalek, J. (2012). Paul Felix Lazarsfeld His Methodological Inspirations and Networking Activities in the Field of Social Research, Prague. Bulletin of Sociological Methodology/Bulletin de Méthodologie Sociologique, 116(1), 61-66.

Kochenkov, A.I. \& Tolstova, Yu.N. (2003) Idea of latent structure Lazersfeld analysis in modern sociology. Sotsiologiya: methodology, methods, mathematical modelirovanie, 16, 125-149.

Muthén, B. \& Asparouhov, T. (2014). Causal effects in mediation modeling: an introduction with applications to latent variables. Structural Equation Modeling: A Multidisciplinary Journal, (ahead-of-print), 1-12.

Maslak, A.A. (2012). Measurement of latent variables in social systems. Slavyansk-on-Kuban: Publishing Center SGPI

Dolnicar, S. \& Leisch, F. (2014). Using graphical statistics to better understand market segmentation solutions. International Journal of Market Research, 56(2), 207-230.

Omelchenko, G.G. (2014). Graph-theoretic modeling of segmentation of market consumers. Economy. Right. Print. Bulletin KSEl. 1 (61), 145-154.

Ponizovkin, D.M. (2011). An optimal graph in a system of collaborative filtering. Software Systems: Theory and Applications, 2(4), 107114.

Adomavicius, G., Sankaranarayanan, R., Sen S. \& Tuzhilin, A. (2005). Incorporating contextual information in recommender systems using a multidimensional approach. ACM Trans. Inf. Syst., 23(1), 103-145.

Tatarova, G.G. (2004). Basis of typological analysis in sociological research: Textbook. M: Publishing house "new textbook".

Keenan, K. F., van Teijlingen, E., \& Pitchforth, E. (2015). The analysis of qualitative research data in family planning and reproductive health care. Journal of Family Planning and Reproductive Health Care, 31(1), 40-43.

de Casterle, B. D., Gastmans, C., Bryon, E., \& Denier, Y. (2012). QUAGOL: A guide for qualitative data analysis. International journal of nursing studies, 49(3), 360-371.

Chenail, R. J. (2012). Conducting Qualitative Data Analysis: Reading Line-by-Line, but Analyzing by Meaningful Qualitative Units. Qualitative Report, 17(1), 266-269.

Kanygin, G.V. (2007). Tools and methodological principles of qualitative data analysis. Sociology: methodology, methods, mathematical models, 25, 70-98.

Martyshenko, N.S. \& Martyshenko, S.N. (2008). Technology to improve the quality of data in the questionnaire. Practical marketing, 1, 813.

Martyshenko, S.N., Martyshenko, N.S. \& Kustov, D.A. (2007). Development Tools typologies according to questionnaires among EXCEL. Academic Journal of Western Siberia, 1, 75-77. 